\title{
Câncer de mama masculino: uma revisão de literatura dos último dez anos
}

Male breast cancer: a literature review of the last ten years

Cáncer de mama masculino: una revisión de literatura de los últimos diez años

Mário Clodoaldo Batista da Costa ${ }^{1 *}$, Stefano do Santos Adorno1, Sebastião Marden Barbosa de Araújoํㅜ, Moisés Castro Saback¹, Kleberson Adriano Silva Gomes¹.

\section{RESUMO}

Objetivo: Revisar na literatura científica sobre o câncer de mama em homens nos últimos dez anos (20072017). Métodos: Desta feita, foi realizado por intermédio da condução de uma revisão bibliográfica básica e sistemática desenvolvida por meio de seleção de artigos em base de dados entre os anos 2007-2017, com o uso dos descritores câncer de mama, homens e incidência. Resultados: Observou-se que os resultados alcançados, com base nos artigos/publicações que existe uma grande influência genética em meio às ocorrências de câncer de mama masculino, como potenciais alterações. A maior frequência de ocorrências da neoplasia ocorre na faixa etária de mais de 60 anos e em indivíduos de cor branca, sendo que o câncer teve mais frequência em meio a Região Sudeste e Sul Conclusão: O câncer de mama é similar em homens e mulheres; todavia, os casos masculinos apresentam particularidades imuno-histoquímicas, mas não existem estudos suficientes para avaliar o impacto dessa característica no prognóstico e tratamento dessa neoplasia.

Palavras-chave: Câncer de mama, Homem, Incidência.

\section{ABSTRACT}

Objectives: To review the scientific literature on breast cancer in men in the last ten years (2007-2017). Methods: This was done through the conduction of a basic and systematic bibliographical review developed through selection of articles in base of data between the years 2007-2017, with the use of breast cancer descriptors, men and incidence. Results: It was observed that the results achieved, based on the articles / publications that there is a great genetic influence in the midst of cancer occurrences of male breast as potential changes. The most frequent occurrence of the neoplasia occurs in the age group of more than 60 years and in individuals of white color, and the cancer was more frequent in the middle of Southeast and South Conclusion: Breast cancer is similar in men and women; however, the male cases present immunohistochemical peculiarities, but there are not enough studies to evaluate the impact of this characteristic on the prognosis and treatment of this neoplasia.

Key words: Breast cancer, Man, Incidence.

\section{RESUMEN}

Objetivo: Revisar en la literatura científica sobre el cáncer de mama en hombres en los últimos diez años (2007-2017). Métodos: De esta manera, fue realizado por intermedio de la conducción de una revisión bibliográfica básica y sistemática desarrollada por medio de selección de artículos en base de datos entre los

1 Universidade Estadual do Amazonas (UEA), Manaus - AM. *E-mail: mcbc_med@hotmail.com 
años 2007-2017, con el uso de los descriptores de cáncer de mama, los hombres y la incidencia. Resultados: Se observó que los resultados alcanzados, con base en los artículos / publicaciones que existe una gran influencia genética en medio de las ocurrencias de cáncer de mama masculino, como posibles alteraciones. La mayor frecuencia de ocurrencias de la neoplasia ocurre en el grupo de edad de más de 60 años y en individuos de color blanco, siendo que el cáncer tuvo más frecuencia en medio a la Región Sudeste y Sur Conclusión: El cáncer de mama es similar en hombres y mujeres; sin embargo, los casos masculinos presentan particularidades inmuno-histoquímicas, pero no existen estudios suficientes para evaluar el impacto de esta característica en el pronóstico y tratamiento de esa neoplasia.

Palabrasclave: Cáncer de mama, Hombre, Incidencia.

\section{INTRODUÇÃO}

Para Philoptts \& Smith (2009), o câncer de mama, como um todo, consiste em uma doença que acomete frequentemente a população feminina mundial e, assim, ocupa, atualmente, o primeiro lugar em incidência, dentre as patologias neoplásicas que acometem caracteristicamente a mulher. Sob esta perspectiva, cabe denotar que, por sua vez, o câncer de mama em indivíduos do sexo masculino é de ocorrência rara e, por isso, não é recorrentemente relatada na literatura.

Estatisticamente para cada 100 novos casos de cânceres mamários femininos, apenas tem-se um caso, ou menos, de câncer masculino, correspondendo à taxa de incidência de $0,8 \%$ até $1,0 \%$ dos casos de câncer mamário que ocorrem mundialmente, ademais, há a observância de que a taxa de mortalidade para os casos de câncer mamário, no geral, refere-se a, aproximadamente, 400 casos de morte por ano (PHILOPTTS \& SMITH, 2009).

O câncer é compreendido como sendo resultado de um erro genético que possibilita a transformação de uma célula normal para a forma de uma célula maligna, sendo categoricamente decorrente de influências hereditárias ou, ainda, ocasionado por agentes físicos/ambientais, biológicos e químicos (CARRARA et al, 2009),em continuidade, compreende-se que o câncer de mama (CM), se diagnosticado precocemente, é uma doença categoricamente tratável, sendo tal descoberta a chave fundamental para que o indivíduo consiga sobreviver à doença (SMOLIN \& MASSIE, 2008).

O câncer geralmente começa com um pequeno nódulo que, com o passar do tempo pode crescer em grandes proporções e, assim, posteriormente, pode se espalhar para áreas nas proximidades das mamas, como nos músculos e na pele, assim como no braço, podendo ainda se espalhar para demais órgãos vitais como fígado, cérebro, pulmão e coluna vertebral (SMOLIN \& MASSIE, 2008).

A baixa procura dos homens pelos serviços de saúde é uma característica recorrentemente e histórica tornando-os mais suscetíveis a desenvolver doenças, estes adiam um tratamento necessário especialmente em função de preconceitos, o que afeta profundamente sua qualidade de vida (AMARAL et al, 2017). A sobrevivência ao câncer coloca-se como uma condição na qual o indivíduo possui a capacidade de conviver e de reconhecer sua condição de ser curado, portanto, a baixa procura dos homens pelos serviços de saúde atinge-os mortalmente (RIESGO et al, 2009).

Especialmente, em relação ao diagnóstico do câncer de mama masculino, importa designar que ainda existe um grande atraso, de forma que, na grande maioria das vezes, a doença é descoberta pelo doente em um estado avançado e, assim, este fato pode estar em atribuição direta em retardo em meio ao processo de diagnóstico (BUSHATSKY et al, 2011).

Com base nestes dimensionamentos, é passível destacar a extrema importância da busca por um diagnóstico do câncer de mama masculino, afinal será nas fases iniciais, em conjunto com a incorporação de condutas e abordagens terapêuticas atualizadas, que poderão ser alcançados os melhores resultados (DANTAS et al, 2014). 
Para Freitas et al (2008), o tratamento assemelha-se profusamente com as recomendações que são aplicáveis ao câncer de mama feminino, mesmo assim, este tratamento é dependente do tipo de tumor, do grau histológico e do acometimento ou não de linfonodos localizados nas axilas, de tal modo, podem encontrar-se associadas modalidades tratamentos específicas, como é o caso da aplicação de cirurgia, radioterapia, terapia hormonal e quimioterapia.

De tal forma, terapia hormonal adjuvante e quimioterapia, utilizando diretrizes semelhantes àquelas aplicadas para mulheres, são recomendados, também, para homens, e assim, entende-se a terapia hormonal como a principal terapia para a doença em estado metástase e, por outro lado, a quimioterapia reserva-se para doença refratária dos hormônios (GIORDANO et al, 2012).

Por fim, e em conformidade com os esclarecimentos evidenciados por Compérat et al (2008), entende-se que o padrão metastático do câncer de mama masculino (CMM) possui semelhanças expressa com a neoplasia que afeta as mulheres em suas mamas, sendo ossos, pulmão e fígado os locais mais comuns para a proliferação da patologia. Ainda segundo os autores as metástases são, geralmente, detectadas de forma incidental, por intermédio do advento da aplicação de exames de imagem para seguimento do tumor primário que seja radiologicamente indistinguível, especialmente se forem percebidas apenas lesões primárias.

\section{MÉTODOS}

Utilizou-se revisão bibliográfica para a promoção de um estudo básico, exploratório e qualitativo fundamentado em artigos científicos entre os anos de 2007 a 2017 e indexados em meio das bases de dados: SciELO, PubMed, ScienceDirect e BIREME, nos quais foram aplicados os descritores: câncer de mama, homens e incidência. No processo de seleção dos artigos utilizou-se critérios de inclusão: estudos com até 10 anos de publicados, e de exclusão: publicado antes de 2007. Sendo selecionados 25 artigos para a elaboração final do trabalho.

\section{RESULTADOS E DISCUSSÃO}

Seguindo as exposições de Silveira et al (2016), há a percepção de que existe uma clara influência genética em meio às ocorrências de câncer de mama masculino, como potenciais alterações em genes supressores BRCA, relações diretas e indiretas com o chamado ponto de checagem CHECK2, histórico familiar e correlações para com a síndrome de Klinefelter, dentre outros fatores específicos.

Acredita-se que a participação do antecedente familiar na etiologia do câncer de mama possa estar relacionada a fatores genéticos, como as mutações de BRCA1/2. Esses genes produzem proteínas que regulam o mecanismo de multiplicação celular e são conhecidos como supressores de tumores. Mutações implicam perda desse controle, e esses genes alterados são transmitidos hereditariamente (PINHO \& COUTINHO, 2007, p. 1067).

Relevante à exposição, tal como expressam os autores Maia et al (2012), de que a chamada síndrome de Klinefelter (SK) é espectro intrinsecamente resultante de uma deficiência genética no cariótipo 47, XXY, podendo levar ao acometimento de hipogonadismo hipergonadotrófico, azoospermia e hipodesenvolvimento dos caracteres sexuais secundários.

Porém, não há entendimento expresso a respeito do mecanismo exato que determine, especificamente, tal deficiência androgênica, sendo variável o grau de disfunção das células de Leydig nos variados casos, ademais, compreende-se que a síndrome de Klinefelter (SK) consiste em uma doença caracteristicamente crônica com sérias repercussões sobre o aparelho reprodutivo masculino, ocasionando significativas causas de infertilidade masculina em nível mundial (Maia et al, 2012).

Silveira et al (2016) ainda complementam a perspectiva de que a síndrome de Klinefelter pode ser relatada como um fator de risco fundamental e importante para o desenvolvimento do câncer de mama masculino, 
especialmente quando a síndrome for influenciada por hormônios, ademais, outras mutações como a CHEK2, CYP17 e no gene supressor tumoral PTEN apresentam menor risco para o acometimento de câncer de mama em homens.

Ademais, os autores Silveira et al (2016) esclarecem que aspectos ambientais também são sugeridos, recorrentemente, pela literatura especializada, frente à manifestação clínica da neoplasia mamária em homens, possuindo, porém, menores ênfases conclusivas.

É enfático que os fatores externos exercem forte influência, tornando relevantes questões a respeito, por exemplo, de riscos ocupacionais, como é o caso dos malefícios potencialmente ocasionados em função de constantes exposições a campos magnéticos ou à radiação, segundo estudo realizado com cidadãos canadenses no ano de 2015 (GRUNDY et al, 2015).

Ainda a respeito de fatores de ordem externa e/ou ambiental, Izuet al (2011) evidenciam que o trabalho noturno pode ser encarado como um fator de risco em meio ao desenvolvimento e ao aparecimento de diversos tipos de cânceres, como por exemplo, de mama, endometrial, de cólon, que são perspectivas recorrentemente demonstradas através de estudos epidemiológicos.

Em continuidade, entende-se que a alteração do ritmo circadiano pela exposição à luz durante a noite pode ser um fator para o acometimento de várias doenças como aquelas de origem gástrica, cardiovascular, desordens no sono e cânceres, e assim, há a percepção de que uma desregulação em meio ao ritmo circadiano pode ocasionar profundas alterações sobre a delicada balança existente entre os fatores promotores e inibidores da divisão celular que levará uma célula normal a se tornar maligna (SILVESTRI et al, 2016; IZU et al, 2011).

Salomon et al. (2015) ainda expõe que cerca de $90 \%$ dos tumores neoplásicos mamários masculinos caracterizam-se por serem ductais invasivos, por outro lado, aproximadamente $80 \%$ apresentam receptores de estrógeno e 80 a 90\% receptores de progesterona efetivamente positivos (NOGUEIRA et al, 2014).

Em estudo com o intuito de comparar e contrastar os cânceres de mama masculino e feminino com base nos dados do Instituto Nacional do Câncer foi possível identificar que a existência de padrões de incidência específicos da idade demonstrou que a biologia do câncer de mama masculino se assemelha expressamente à do câncer de mama feminino com início tardio (ANDERSON et al, 2010; MOTA, 2010).

Ademais, tendências semelhantes de incidência de câncer de mama entre homens e mulheres sugerem acerca da existência de fatores comuns de risco de câncer de mama para ambos os sexos, especialmente a perspectiva de que o câncer de mama tanto em homens quanto em mulheres é positivo ao receptor de estrogênio (ANDERSON et al, 2010; MOTA, 2010).

E por fim, Anderson et al (2010) e Mota (2010) trazem ainda a evidência de que mortalidade por câncer de mama e as taxas de sobrevivência podem melhorar significativamente ao passar do tempo para o câncer de mama masculino e feminino, porém, categoricamente o progresso para os homens ficou atrás dos avanços observados nas mulheres.

No que tange à diagnose das neoplasias mamárias masculinos, entende-se que o autoexame é o maior aliado dos pacientes para o alcance de uma detecção precoce (DANTAS et al, 2015), ou seja, o câncer de mama em homens necessariamente exige a tomada de medidas preventivas, dentre elas, encontra-se a realização de exames cito e histopatológicos (DANTAS et al, 2014), ademais, deve ser de entendimento consagrado à perspectiva de que a prevenção e o diagnóstico precisos são as melhores estratégias para 0 alcance de melhorias na qualidade de vida e na sobrevivência do paciente com esta neoplasia específica (HAAS et al, 2009).

A autopalpação ou autoexame da mama é importante para a detecção precoce da patologia. O homem deve realizar o autoexame uma vez ao mês, seguindo os mesmos passos orientados às mulheres, agendando um dia do mês para a realização do autoexame (SOUZA et al, 2017, p. 10). 
Com base nestas disposições, entende-se como sendo eminentemente necessário proporcionar abordagens e atitudes que possam conscientizar a população masculina acerca do fato de que o autoexame é um importante instrumento para alcançar um diagnóstico precoce das neoplasias mamárias e, assim, o autoexame deve ser realizado mensalmente e, consequentemente, o auxílio médico deverá ser rapidamente procurado quando em casos de alteração mamária ao toque (SOUZA et al, 2017).

Espinola et al (2013) ainda dispõem que devem ser viabilizados como forma de complementação diagnóstica os exames de imagem, geralmente com o uso de mamografia associada ou não e, também, de ultrassonografia das mamas, com a finalidade de dispor de um diagnóstico diferencial para com demais patologias benignas que se assemelham à neoplasia mamária, como a ginecomastia, lipomas, ou até mesmo para a descoberta precoce de metástase de outros carcinomas para a mama.

Por outro lado, é necessário enfatizar que os fatores prognósticos do câncer de mama em homens compreendem o tamanho tumoral, o grau histológico e o comprometimento linfonodal, ou seja, a neoplasia analisada é similar entre homens e mulheres; entretanto, indivíduos do sexo masculino apresentam particularidades imuno-histoquímicas específicas, porém, na atualidade não existem estudos suficientes para que seja possível dispor a respeito de uma avaliação do impacto de tal característica no prognóstico e no tratamento dessa neoplasia (NOGUEIRA et al, 2014).

Salomon et al (2015) e Nogueira et al (2014) elucidam ainda que o tratamento para homens com neoplasia mamária extrapola os estudos sobre câncer de mama em mulheres, e assim, podem ser empregadas às abordagens de cirurgia, hormonioterapia, quimioterapia e radioterapia, utilizadas em conformidade com os guidelines femininos, utilização que, segundo Riesgo et al (2009), se dá em função da característica raridade da patologia, reforçando a necessidade de conscientização da população, afinal, o câncer de mama masculino deve ser compreendido como um diagnóstico possível aos homens.

Falta de conscientização e preconceito por parte dos homens da importância da detecção do câncer de mama tem sido considerada a principal causa de óbito. A procura do diagnóstico e tratamento precoce do câncer de mama poderá acelerar o passo do Brasil no sentido de aumentar a sobrevida dos homens com câncer de mama. Embora os números não sejam alarmantes, um pouco de informação e menos preconceito ajudariam a evitar sofrimento e até mortes (CUNHA FILHO et al., 2012, p. 16).

O desenvolvimento de terapia hormonal adjuvante e quimioterapia, com a aplicação das mesmas diretrizes para as mulheres, são recomendados para homens com câncer de mama, além disso, há a observância de que a terapia hormonal compreende o principal caminho tratamental para a doença em fase metastática; e, por sua vez, entende-se que a quimioterapia deve ser especialmente reservada para a doença refratária dos hormônios (GIORDANO et al, 2012).

Para Souza \& Vieira (2013) o tratamento fisioterapêutico após a realização de retiradas de tumores e mastectomias é fundamental e se mostra bastante eficaz, proporcionando melhorias na amplitude de movimento, na força muscular, compreendendo redução da região do edema e promovendo o alcance de independência funcional por parte do paciente em meio à realização das suas atividades cotidianas e corriqueiras.

Por tais razões oportunas, é evidentemente necessária à condução de um acompanhamento fisioterapêutico mediante o pós-operatório de mastectomias, o seu intencionamento central refere-se a minimizar as complicações que decorrem caracteristicamente de tal procedimento, potencialmente melhorando a qualidade de vida dos pacientes, em especial dos indivíduos que são acometidos pelo câncer em idade avançada que, em geral, é uma população que possui maior fragilidade no que diz respeito à saúde (SOUSA \& VIEIRA, 2013).

A respeito da incidência do câncer de mama masculino, que é o objeto central desta pesquisa, Haas et al (2009) mencionam que a mesma aumentou, significativamente, de 0,86 a 1,06 para cada 100.000 homens no decorrer dos últimos 26 anos; de forma que as taxas mais altas ocorrem na África (5\% a 15\%), na América do Norte e na Europa e, por sua vez, as taxas mais baixas concentram-se na Ásia. No Amazonas, no período 
de 2007 a 2015 foram registrados 25 casos pela Fundação Centro de Controle de Oncologia do Amazonas (FCECON, 2017).

Haas et al (2009) ainda comenta a respeito do fato de que a incidência da neoplasia mamária em homens varia profusamente entre os países, e assim, a exemplo, esta forma de câncer representa cerca de $1,2 \%$ dos recém-diagnosticados nos Estados Unidos e cerca de 1\% de todos os cânceres de mama que acometem habitantes do continente europeu.

Complementando acepções, Mota (2010) evidencia que as diferenças de incidência entre zonas geográficas assemelham-se diretamente aos casos das mulheres com câncer da mama, comportando-se como mais frequente na América do Norte, na África e na Europa e, por sua vez, apresentando menor frequência na Ásia.

As altas taxas de incidência observadas na África estão relacionadas com o elevado número de doenças infecciosas endêmicas que assolam as populações de tal continente - como, por exemplo: hepatites B e C, e bilharziose (esquistossomose) -, levando ao desenvolvimento de lesões crônicas no fígado, dando origem, geralmente, a uma condição de hiperestrogenismo (MOTA, 2010).

Importante denotar ainda que a hepatite viral refere-se a uma inflamação do fígado que pode ser provocada por um dos cinco vírus distintos da hepatite $(A, B, C, D$ e $E)$, sendo relevante a demonstração de que os vírus da hepatite $A$ e $E$ pode ser transmitidos pela via fecal-oral, os das hepatites $B$ e $C$ são transmitidos pela exposição ao sangue, relações sexuais e, também, nos casos em que uma mãe infectada passa a afecção para o seu feto. Destarte, embora também possa ser transmitida pelo sangue, a hepatite $D$ apenas pode ocasionar uma forma de infecção em pessoas com infecção por hepatite $B$ ativa ou em portadores do vírus (Oms, 2016).

Categoricamente, a hepatite viral deve ser entendida sob a forma de um problema de saúde pública especificamente endêmico em meio à região Africana, podendo ser comparada a outras doenças transmissíveis graves, como por exemplo, o $\mathrm{VIH}$, a tuberculose e o paludismo, tal como expressam os dados oficiais da Organização Mundial da Saúde (Oms, 2016).

De forma complementar, é fundamental a exposição de que todos os cinco vírus da hepatite $(A, B, C, D$, E) podem ocasionar a doença em sua fase mais aguda, mas os números mais elevados de morte resultam expressamente de cânceres de fígado e de problemas clínicos-patológicos, como é o caso da cirrose, problemáticas estas que ocorrem ao decorrer de décadas de infecção por hepatite crônica do tipo B ou C (OMS, 2016).

Os autores Dantas et al (2016), tomando por base os dados coletados no site do DataSUS, esclarecem acerca do entendimento da incidência do câncer de mama em homens em território brasileiro, sendo assim, observam-se maior frequência de ocorrências da neoplasia na faixa etária de mais de 60 anos e em indivíduos de cor branca, ademais, o câncer é mais frequente em meio a Região Sudeste e Sul.

De forma geral, os autores ainda demonstraram a necessidade de transformações sobre as práticas de cuidados médicos que são prestadas ao homem para que, então, os mesmos passem a tomar mais consciência, compreendendo que também possuem mamas e, portanto, podem desenvolver o câncer mamário (DANTAS et al, 2016).Complementarmente, os autores ainda denotam a perspectiva de que:

Regiões Sudeste e Sul têm apresentado valores de grande significância quando relatado casos de internações e óbitos por $\mathrm{CAMH}$, revelando a importância de todo o território brasileiro se mobilizar no sentido de fortalecer as políticas públicas voltadas para populaçã e as práticas de prevenção para o câncer de mama, que em nossa realidade estão voltadas apenas para o grupo feminino (DANTAS et al., 2016, p. 33).

Neste ínterim, Marques e Júlio (2012) ainda demonstram que é necessário o alcance de uma maior conscientização por parte do público masculino, especialmente no que diz respeito ao âmbito da saúde, e que, assim, os homens passem a buscar em maior recorrência e constância pelos serviços de saúde, realizando exames com a finalidade expressa de evitar que os mesmos se tornem estatísticas das vítimas 
fatais ou do diagnóstico tardio de um câncer de mama, ou seja, e tal como esclarecem Vieiro et al (2011), o desconhecimento sobre a patologia pode ser considerado enquanto um enfático fator de risco.

Em meio à condução de um estudo retrospectivo, Espinola et al (2013) concluem a respeito da existência da pouca informação sobre o câncer de mama masculino, em especial pelo fato de que os pacientes do sexo masculino, geralmente, compreendem uma demora para que procure por avaliação médica adequada, pressuposto que pode ocasionar críticos atrasos no diagnóstico, além da descoberta da patologia já em estágios clínicos avançados, circunstância que pode impactar negativamente na sobrevida do paciente.

Schraiber et al (2010) depreendem que a atenção primária ainda é especificamente voltada ao dimensionamento de cuidados em saúde para as mulheres, situação que se reproduz em meio ao funcionamento dos serviços e no que tange aos desempenhos profissionais, estabelecendo a presença de desigualdades de gênero, afinal, há para as mulheres a disciplina do cuidado e, por outro lado, para os homens, observam-se recorrentes impropriedades para a disposição de assistência e cuidado.

Complementando as observações elucidadas anteriormente, é necessário dispor ainda que:

A população deve realizar exames rotineiros e levar uma vida saudável, o que pode impedir o acometimento ou diminuir o comprometimento dessa neoplasia assim como de várias outras enfermidades. A visão preconceituosa para essa neoplasia apenas a torna mais abrupta. Portanto a sociedade tem que passar a ser mais informada de certos tipos de cânceres que são raros, como o tratado nessa pesquisa (MARQUES E JÚLIO, 2012, p. 161).

Corroborando com estes aspectos, Toneli et al (2010) trazem a percepção de que a categoria gênero refere-se a um elemento de grande importância frente ao padrão dos riscos de saúde nos homens e, também, na forma como estes podem ser perceber, utilizar e cuidar de seus corpos, tanto como possibilidade de transformação de valores como fator potencial em meio à reiteração dos problemas de saúde vigentes.

\section{CONCLUSÃO}

Observou-se que inexistem políticas públicas e manuais relacionados ao manejo e tratamento do câncer de mama masculino, tal circunstância decorre da baixa incidência de tal patologia associada com 0 preconceito que existe por parte dos homens em relação à condição que apresenta, sendo ainda frágeis e incipientes os esforços para o desenvolvimento de políticas públicas, com predisposição ao acometimento de câncer de mama A análise dos fatores genéticos BRCA1/2 é elemento recorrente na literatura especializada, e assim, é imprescindível, em especial pelo fato de que os referidos fatores exibem as características patológicas distintas e mais eficazes ao diagnóstico do câncer de mama em comparação com os hemogramas totais.

\section{REFERÊNCIAS}

1. Amaral DED, et al. Câncer de mama masculino: o contexto do sobrevivente. Rev. enferm UFPE online (Internet), Recife;11(5):1783-90, mai. 2017.

2. BUSHATSKY M, BARROS MBSC, Interaminense INCS, Rosendo PG, Beltrão Neto JE, FIGUEIRA FILHO ASS. Câncer de mama masculino: estudo de caso em dois serviços especializados da cidade do recife, Brasil. Ver. enferm UFPE online [Internet];5(4):951-56, jun. 2011.

3. CARRARA S, et al. A política de atenção à saúde do homem no Brasil: os paradoxos da medicalização do corpo masculino. Physis [online]. 2009; 19(3):659-678.

4. COMPÉRAT E, et al. Splenic metastases: clinic pathologic presentation, differential diagnosis, and pathogenesis. Archivesof Pathology and Laboratorial Medicine; 2008;131:965-9.

5. CUNHA FILHO PMC, et al. Câncer de mama masculino: prevalência, aspectos epidemiológicos, clínicos, terapêutico e assistência de enfermagem. Revista In Saúde, Faculdade Santa Emília de Rodat, jun. 2012;3(9)1217.

6. DANTAS RCO, et al. Câncer de mama em homem: uma realidade brasileira. REBES - Revista Brasileira de Educação e Saúde, Pombal, jul./set. 2015;5(3):29-34. 
7. DANTAS RCO, et al. Ocorrência de neoplasias mamaria no homem do nordeste brasileiro. In: I Congresso Nacional de Ciências da Saúde. Avanços, Interações e Práticas Integrativas. Cajazeiras: CONACIS, 2014.

8. ESPINOLA JP, et al. Câncer de mama masculino: análise de 12 casos em uma única instituição. Revista Brasileira de Mastologia, 2013;23(3):87-91.

9. FCECON. Relatório Anual de Gestão. Disponível em: http://www.fcecon.am.gov.br/institucional/acesso-ainformacao/relatorios-de-gestao/.

10. FREITAS MAS, et al. Perfil imunohistoquímico de carcinomas mamários invasores em homens. J. Bras. Patol. Med. Lab. [online]. 2008,44(5):375-380GIORDANO et al, 2012

11. GIORDANO SH, et al. Câncer de mama nos homens. Ann Intern Med;137(8):678-87, out. 2012.

12. GRUNDY A, et al. Occupational exposure to magnetic fields and breast cancer among Canadian men. Cancer Medicine, 2015;5(3):586 -596. Haas et al (2009

13. HAAS P, et al. Epidemiologia do câncer de mama em homens. Revista do Instituto Adolfo Lutz, São Paulo;68(3), 2009

14. IZU M, et al. Trabalho noturno como fator de risco na carcinogênese. Ciencia y enfermería, dez. 2011;17(3):8395.

15. MAIA FFR, Diagnóstico Tardio da Síndrome de Klinefelter - Relato de Caso. Arquivos Brasileiros de Endocrinologia e Metabolismo, jun. 2012;46(3):306-309.

16. MARQUES DL JÚLIO ICF. Câncer de Mama Masculino: uma revisão sistemática. Revista UNINGÁ, Maringá PR, out./dez. 2012;34:155-162.Mota (2010

17. NOGUEIRA SP, et al. Câncer de mama em homens. Rev Bras Mastologia;24(4):109-114, 2014.

18. OMS - Organização Mundial da Saúde. Prevenção, cuidados e tratamento da hepatite viral na região africana: quadro de acção, 2016 - 2020. Comitê Regional para a África. 67ª sessão. República Federal Democrática da Etiópia. Organização Mundial da Saúde. Etiópia: Oms, 2016.

19. PHILOPTTS LE, SMITH RA. Screening for breast cancer. Sem (in) Roentgenology. 2009;38(1):19-33.PINHO\& COUTINHO, 2007, p. 1067).

20. RIESGO IS, et al. Câncer de mama em homem: relato de caso e revisão da literatura. Rev AMRIGS [Internet];53(2):198-201, abr./jun. 2009Salomon et al (2015

21. SALOMON MFB, et al. Câncer de mama no homem. Revista Brasileira de Mastologia, jan. 2015;25(4):141-145.

22. SCHRAIBER LB, et al. Necessidades de saúde e masculinidades: atenção primária no cuidado aos homens. Cad saúde pública [Internet], mai. 2010;26

23. SILVEIRA NMT. Fatores genéticos associados ao câncer de mama masculino: uma revisão de literatura. Jornal Interdisciplinar de Biociências, 2016;1(2):27-30.

24. SILVESTRI V, et al. Male breast cancer in BRCA1 and BRCA2 mutation carriers: pathology data from the Consortium of Investigator sof Modifiers of BRCA1/2. Breast Cancer Research, 2016;18:15.

25. SMOLIN Y, Massie MJ. Male breast cancer: a review of the literature and a case report. Psychosomatics. 2008;43(4):326-30

26. SOUZA CF, VIEIRA MCA. Tratamento fisioterapêutico no câncer de mama masculino: relato de caso de um idoso acometido por esta rara patologia. In: III CiEh - Congresso Internacional de Envelhecimento Humano. Avanços da ciência e das políticas públicas para o envelhecimento. Campina Grande: CIEH, 2013.

27. TONELI MJF, et al. Masculinidades e práticas de saúde: retratos da experiência de pesquisa em Florianópolis. Physis (Rio J.) [Internet]. 2010;20(3):973-94.

28. VIERO V, Machado BP, Gebert L, Oliveira MD, Lima SBS, Ferreira TF. Incidência de câncer de mama na população masculina de um hospital universitário. Centro Universitário Franciscano. Unifra, 2011. 